Tome 42, no 23. - Octobre 1935.

\title{
Etude des Strigéidés de la collection du Département de Parasitologie de l'Université Hébraïque de Jérusalem
}

par

\section{Georges DUBOIS}

Docteur ès sciences.

(Travail de l'Institut de Zoologie de l’Université de Neuchâtel.)

Avec 7 figures dans le texte.

Le matériel ayant permis l'étude des formes nouvelles décrites dans les pages qui suivent, provient de la Collection helminthologique du Département de Parasitologie de l'Université Hébraïque, à Jérusalem.

Recueilli en Syrie, à Kirik-Khan, en mars et avril 1930, il fut obligeamment mis à notre disposition par M. le Professeur $\mathrm{D}^{\mathrm{r}} \mathrm{G}$. Witenberg, auquel nous exprimons ici nos sincères remerciements.

Les diagnoses originales de ces parasites ont été publiées dans les "Actes de la Société Helvétique des Sciences Naturelles", Zurich, 1934, p. 374-375.

\section{Apharyngostrigea flexilis Dubois, 1934.}

Famille: Strigeidae Railliet, 1919.

Sous-famille: Strigeinae Railliet, 1919.

Nous n'avons reçu que quatre exemplaires de ce parasite; ils ont été montés en préparations totales pour l'examen morphologique, puis les deux exemplaires les mieux conservés (dont un est le type) ont été débités en coupes en vue de l'étude anatomique. Nous n'avons trouvé aucune trace de pharynx; la ventouse buccale elle-même n'était visible que sur les coupes. Nous rattachons

Rev. Suisse de Zool., T. 42, 1935. 
donc ${ }^{1}$ cette espèce au genre Apharyngostrigea Ciurea, 1927, qui jusqu'à présent ne comptait que des parasites d'Ardeidae.

Le matériel provient de l'intestin de Circus macrurus (Gm.) [syn. Circus swainsoni Smith], collection du Département de Parasitologie de l'Université

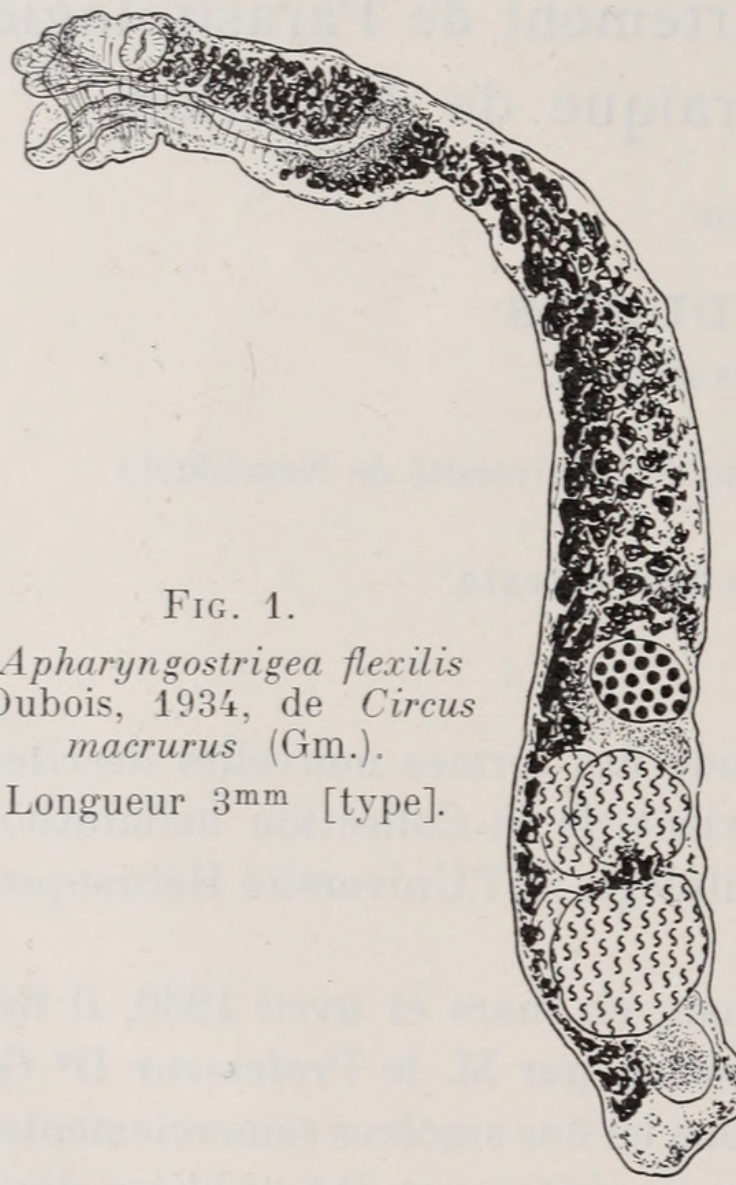
Hébraïque de Jérusalem, tube $n^{0}$ 265, étiqueté "Strigeidae sp. ». Il a été récolté à KirikKhan, en Syrie, le 8 avril 1930.

\section{Description .}

La partie antérieure est très allongée (2 à 3 fois plus longue que large), utriforme.

La partie postérieure est également allongée, cylindrique ou claviforme, souvent un peu renflée dans sa seconde moitié, au niveau des testicules, tandis que la première moitié, formant la région du cou (Halsteil), diminue progressivement de diamètre vers l'avant. I'axe longitudinal est rectiligne ou faiblement incurvé. A son extrémité postérieure, le corps est tronqué perpendiculairement à l'axe longitudinal. Le pore génital est terminal.

Longueur totale: jusqu'à $3 \mathrm{~mm}, 5$.

\section{Longueur}

Partie antérieure: $\quad 0 \mathrm{~mm}, 84-0 \mathrm{~mm}, 90$

Partie postérieure: $1 \mathrm{~mm}, 90-2 \mathrm{~mm}, 70$

\section{Largeur}

0mm, 30-0mm, 42 .

$0 \mathrm{~mm}, 35-0 \mathrm{~mm}, 51$.

Rapport $\frac{\text { Longueur de la partie postérieure }}{\text { Longueur de la partie antérieure }}=2,11$ à 3,21 .

Rapport $\frac{\text { Longueur de la partie antérieure }}{\text { Largeur de la partie antérieure }}=2$ à 3 .

1 Sous réserve d'observations ultérieures. 


$$
\text { Rapport } \frac{\text { Longueur de la partie postérieure }}{\text { Largeur de la partie postérieure }}=4,22 \text { à 5,68. }
$$

Les ventouses sont très faiblement développées, difficilement visibles sur les préparations totales et très rapprochées l'une de l'autre. Leurs diamètres sont:

Ventouse buccale: $50 / 60 \mu$ (visible sur les coupes).

Ventouse ventrale: $90-95 / 63-90 \mu$.

La ventouse ventrale est située des 23/100 aux 28/100 de la longueur de la partie antérieure. (Elle est seule visible sur la figure 1).

Les lobes de l'organe tribocytique (adhésif) font saillie à l'extrémité antérieure. La glande de cet organe est ovoïde; elle se trouve dans le dernier tiers de la partie antérieure du corps et mesure environ $100 \mu$ sur $60 \mu$.

Les glandes génitales sont situées dans la deuxième moitié de la partie postérieure du corps.

L'ovaire, ellipsoïdal, est situé entre les 50/100 et les 58/100 de la longueur de cette partie du corps; il est tangent au premier testicule et mesure 180 à $235 \mu$ sur 140 à $160 \mu$.

La glande de Mehlis et le réservoir vitellin se trouvent entre les deux testicules. La branche ascendante de l'utérus remonte assez loin antérieurement, dans la région du cou où elle s'incurve brusquement pour redescendre ventralement, suivant la ligne médiane, jusqu'à l'extrémité distale où s'ouvre le pore génital terminal.

Les œufs mesurent 103 à $108 \mu$ sur 60 à $72 \mu$.

Les follicules vitellogènes s'étendent dans les deux parties du corps, du niveau de la ventouse ventrale, à travers toute la partie postérieure, jusqu'à 90 à $135 \mu$ de l'extrémité postérieure. Ils sont surtout abondants dans la région du cou.

Les testicules sont massifs, bilobés et incurvés en forme de fer à cheval. Ils mesurent 270 à $460 \mu$ de diamètre transversal et 150 à $360 \mu$ de diamètre longitudinal. Dans l'espace délimité par leur concavité ventrale s'abritent l'utérus et les conduits génitaux mâles. La vésicule séminale est située dans la concavité postérieure du deuxième testicule. Le bord antérieur du premier testicule se trouve entre les 57/100 et les 62/100 de la longueur de la partie postérieure du corps.

Collection. - Le type et les paratypes sont déposés dans notre collection particulière. 


\section{Cotylurus hebraicus Dubois, 1934.}

Famille: Strigeidae Railliet, 1919.

Sous-famille: Strigeinae Railliet, 1919.

Ce parasite provient de l'intestin de Fulica atra L. [Collection du Département de Parasitologie de l'Université Hébraïque de Jérusalem, tube no 261; matériel récolté à Kirik-Khan (Syrie), le 8 avril 1930 et étiqueté «Strigeidae sp.»].

\section{Description.}

La partie antérieure du corps est en forme de coupe globulaire, tronquée obliquement, ce qui fait que la paroi dorsale est beaucoup plus longue que la paroi ventrale. L'axe de la partie postérieure est faiblement arqué. Le pore génital est dorsal, subterminal.

Longueur totale: jusqu'à $2 \mathrm{~mm}, 4$.

\section{Longueur}

Partie antérieure:

Partie postérieure: $0^{\mathrm{mm}}, 54-0 \mathrm{~mm}, 68$ $1^{\mathrm{mm}}, 28-1^{\mathrm{mm}}, 65$
Largeur

$$
0^{\mathrm{mm}}, 52-0^{\mathrm{mm}}, 62 \text {. }
$$$$
0^{\mathrm{mm}}, 51-0^{\mathrm{mm}}, 65 \text {. }
$$

$$
\text { Rapport } \frac{\text { Longueur de la partie postérieure }}{\text { Longueur de la partie antérieure }}=2,37 \text { à } 2,46 \text {. }
$$

La ventouse buccale est située tout à fait au bord de la coupe que forme la partie antérieure. La ventouse ventrale se trouve entre les 40/100 et les 53/100 de la longueur de la partie antérieure, donc assez rapprochée de la ventouse buccale.

Les diamètres des ventouses sont:

Ventouse buccale: 100 à $135 \mu$ sur 95 à $118 \mu$.

Ventouse ventrale: 150 à $180 \mu$ sur 125 à $153 \mu$.

L'examen des coupes a révélé l'existence d'un pharynx très petit, mesurant 43 à $45 \mu$ de longueur sur 31 à $35 \mu$ de largeur et qui n'était pas visible sur les préparations totales. Les caeca atteignent presque l'extrémité postérieure du corps: ils se terminent au niveau du fond de la bourse copulatrice.

L'ovaire, ellipsoïdal, mesure 140 à $190 \mu$ sur 110 à $160 \mu$. Il est situé entre les 18/100 et les 20/100 de la longueur de la partie postérieure du corps. La glande de Mehlis et le réservoir vitellin se trouvent 
entre les deux testicules. L'utérus s'étend sur toute la longueur de la partie postérieure du corps; sa branche descendante est située ventralement. Dans la partie terminale, elle se recourbe en arrière du deuxième testicule et de la vésicule séminale pour traverser le corps suivant une direction dorso-ventrale et venir déboucher, avec le canal éjaculateur, au fond de l'atrium génital, du côté dorsal.

Les œufs mesurent 86 à $90 \mu$ sur 55 à $65 \mu$.

Les follicules vitellogènes occupent toute la partie postérieure où ils s'étendent jusqu'à l'atrium génital, sans masquer ce dernier. Quelques rares follicules pénètrent dans la partie antérieure où ils sont disséminés jusqu'au niveau de la ventouse ventrale.

Les testicules sont recourbés en forme de fer à cheval; leurs faces dorsale et antérieure sont convexes, tandis que leurs faces ventrale et postérieure sont concaves, creusées par un profond sillon médian. Il en résulte que, sur les coupes, les masses testiculaires apparaissent bilobées, réniformes ou cordiformes, à bords entiers. Leurs dimensions, suivant les différents axes, oscillent entre $240 \mu$ et $400 \mu$. Le bord antérieur du premier testicule est situé entre les 22/100 et les 28/100

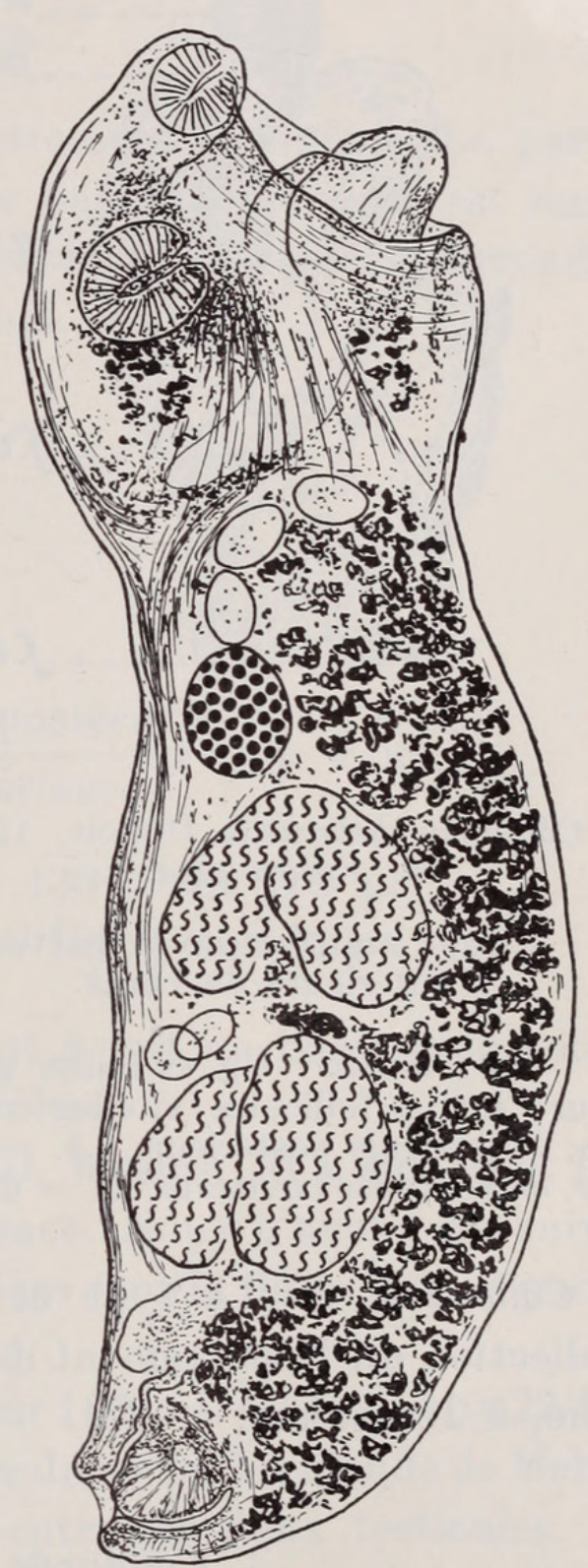

FIg. 2.

Cotylurus hebraicus Dubois, 1934, de Fulica atra L.

Longueur $1^{\mathrm{mm}}, 82$ [type]. de la longueur de la partie postérieure du corps. La vésicule séminale est grande et sinueuse; elle se prolonge par un canal éjaculateur venant déboucher avec l'utérus au fond de l'atrium génital, sur le côté dorsal. 
Le bulbe musculeux occupe à peu près toute la cavité de la bourse copulatrice dont l'ouverture est dorsale, subterminale.

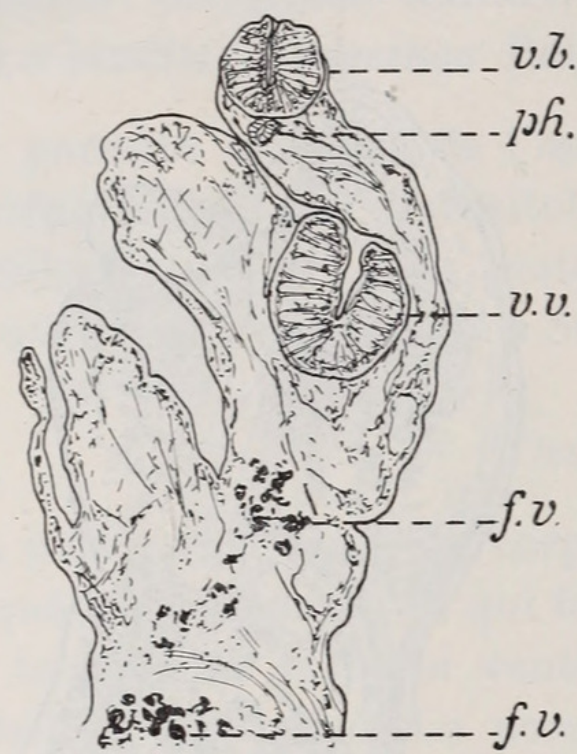

FIG. 3.

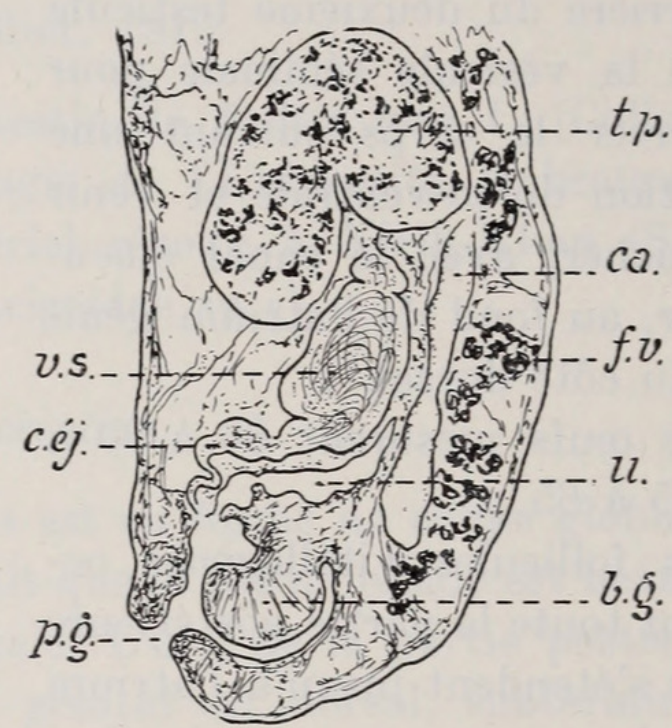

FIG. 4.
Cotylurus hebraicus Dubois, 1934, de Fulica atra L.

Coupe sagittale de la partie antérieure du corps.
Cotylurus hebraicus Dubois, 1934, de Fulica atra L.

Coupe sagittale de la seconde moitié de la partie postérieure du corps.

Abréviations: $b . g .=$ bulbe génital; $c a .=$ caeca; $c . e ́ j .=$ canal éjaculateur; $f .{ } .=$ follicules vitellogènes; $p . g .=$ pore génital; $p h .=$ pharynx; t.p. = testicule postérieur $; \bullet . b .=$ ventouse buccale; $\bullet . s .=$ vésicule séminale; o.o. $=$ ventouse ventrale; $u .=$ utérus.

Collection. - Le type et les paratypes sont déposés dans la collection du Département de Parasitologie de l'Université Hébraïque, à Jérusalem, no 261.

\section{Cotylurus syrius Dubois, 1934.}

Famille: Strigeidae Railliet, 1919.

Sous-famille: Strigeinae Railliet, 1919.

La description de cette espèce est basée sur l'examen d'un seul exemplaire, provenant de l'intestin de Mareca penelope (L.), monté tout d'abord en préparation totale et que représente la figure 5, puis débité en coupes (fig. 6) en vue de l'étude anatomique. Le matériel fait partie de la collection helminthologique du Dépar- 
tement de Parasitologie de l'Université Hébraïque, à Jésusalem [tube no 279]; il a été récolté à Kirik-Khan (Syrie), le 10 avril 1930 et étiqueté "Strigeidae sp.».

\section{Description.}

Les deux parties du corps sont nettement distinctes. La partie antérieure est arrondie, globulaire; la partie postérieure est sacciforme, un peu arquée, à extrémité postérieure largement arrondie. Le pore génital est dorsal, subterminal.

Longueur totale: $2 \mathrm{~mm}, 085$.

$\begin{array}{llr} & \text { Longueur } & \text { Largeur } \\ \text { Partie antérieure: } & 0 \mathrm{~mm}, 585 & 0 \mathrm{~mm}, 675 . \\ \text { Partie postérieure: } & 1 \mathrm{~mm}, 5 & 0 \mathrm{~mm}, 570 .\end{array}$

Rapport $\frac{\text { Longueur de la partie postérieure }}{\text { Longueur de la partie antérieure }}=2,56$.

Diamètre de la ventouse buccale: $130 \mu$ sur $105 \mu$.

Diamètre de la ventouse ventrale: $180 \mu$ sur $160 \mu$.

La ventouse buccale est située tout à fait au bord de la coupe que forme la partie antérieure.

Le pharynx, très petit et ellipsoïdal, mesure $70 \mu$ sur $40 \mu$. Les coupes seules en ont révélé l'existence. Nous n'avons pu suivre le tracé des caeca que dans la première moitié de la partie postérieure du corps.

L'ovaire, ellipsoïdal, mesure $153 \mu$ sur $140 \mu$. Il est situé aux 22/100 de la longueur de la partie postérieure du corps. La glande de Mehlis et le réservoir vitellin se trouvent entre les deux testicules. La branche ascendante de l'utérus remonte jusqu'au niveau de la constriction séparant les deux parties du corps; la branche descendante suit la face ventrale; dans sa partie terminale, elle se recourbe en arrière du deuxième testicule et de la vésicule séminale pour traverser le corps suivant une direction dorso-ventrale et vient déboucher, avec le canal éjaculateur, au fond de l'atrium génital, du côté dorsal. Les œufs sont particulièrement grands, ce qui constitue l'un des caractères spécifiques. Ils mesurent 120 à $137 \mu$ sur 68 à $84 \mu$. 
Les follicules vitellogènes sont confinés uniquement dans la partie postérieure du corps où ils s'étendent presque jusqu'à l'extrémité arrondie, laissant à découvert l'atrium génital. Aucun ne pénètre dans la partie antérieure.

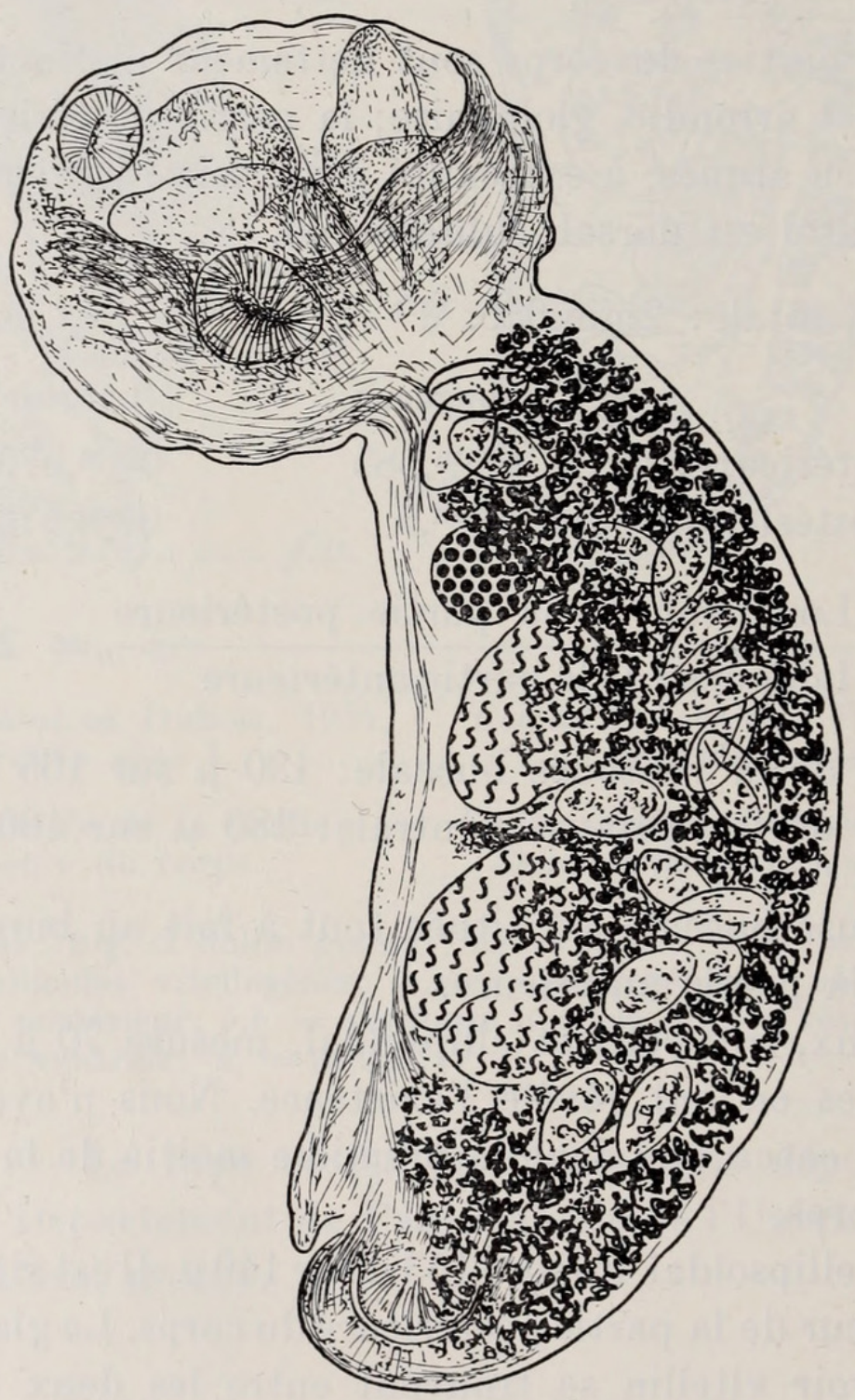

FIG. 5.

Cotylurus syrius Dubois, 1934, de Mareca penelope (L.).

Longueur $2^{\mathrm{mm}}, 085$ [type].

Sur la préparation totale, les testicules apparaissaient massifs, plus ou moins sphériques ou cordiformes, à bords entiers; ils mesuraient suivant les axes 270 à $380 \mu$. En réalité, et d'après les coupes, ils sont légèrement recourbés en forme de fer à cheval, 
c'est-à-dire que chacun d'eux présente deux masses principales, l'une à gauche et l'autre à droite du plan médian, réunies par une partie commissurale plus étroite et située dorsalement. L'échancrure médiane et ventrale du deuxièrne testicule se continue sur la face postérieure de cet organe où elle délimite l'espace occupé par la vésicule séminale. Le bord antérieur du premier testicule est situé aux 26/100 de la longueur de la partie postérieure du corps. La vésicule séminale est grande; sa partie principale s'abrite

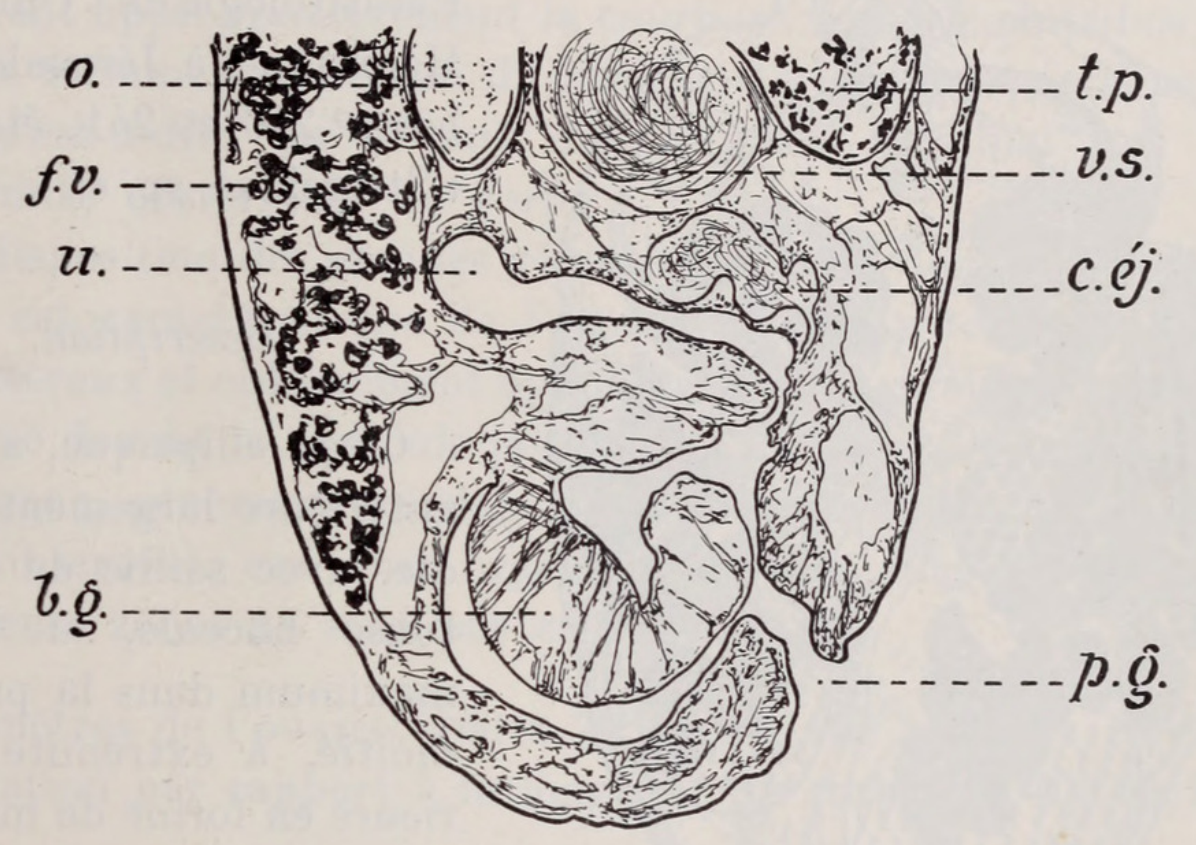

FIG. 6.

Cotylurus syrius Dubois, 1934, de Mareca penelope (L.). Coupe sagittale de l'extrémité postérieure du corps. $o$. = œuf; pour les autres abréviations voir fig. 3 et 4 .

partiellement dans l'échancrure de la face postérieure du deuxième testicule; les parois de la partie terminale sont musculaires; le canal éjaculateur vient déboucher, avec l'utérus, au fond de l'atrium génital, du côté dorsal.

Le bulbe musculeux occupe à peu près toute la cavité de la bourse copulatrice dont l'ouverture est dorsale, subterminale.

Collection. - L'exemplaire unique (en coupes) est déposé dans la collection du Département de Parasitologie de l'Université Hébraïque, à Jérusalem, no 279. 
4. Prohemistomum syriacum Dubois, 1934.

Famille: Cyathocotylidae Poche, 1925 [= Cyathocotyleae Mühling, 1896].

L'hôte de ce parasite est la Cigogne blanche, Ciconia ciconia (L.) [intestin]. Il en a été recueilli 7 exemplaires, à Kirik-Khan, en

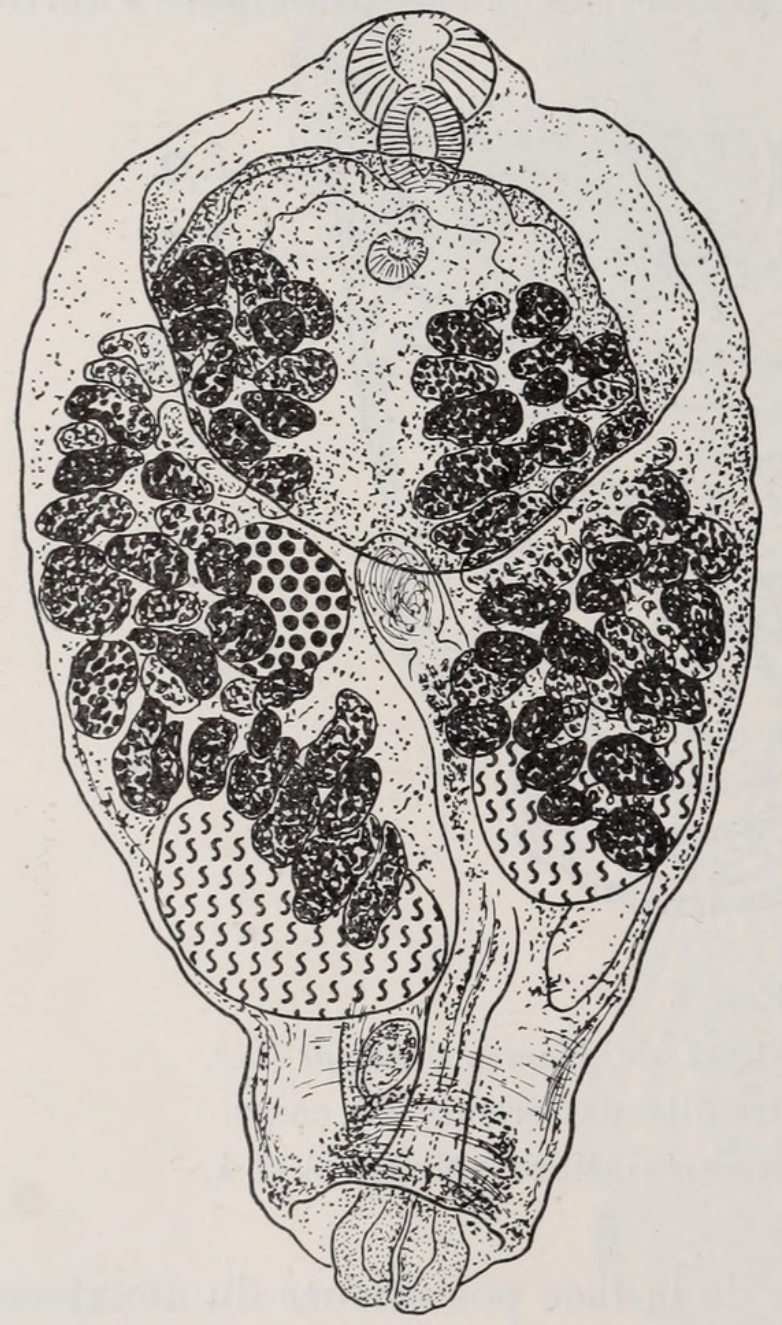

FIG. 7. Syrie, le 30 mars 1930 [Collection du Département de Parasitologie de l'Université Hébraïque, à Jérusalem, tubes $n^{\circ} 240$ et 241 , étiquetés «Strigeidae sp.»].

\section{Description.}

Corps elliptique, à région antérieure largement arrondie, avec saillie de la ventouse buccale, à largeur maximum dans la première moitié, à extrémité postérieure en forme de manchon court, à organe tribocytique (adhésif) volumineux, largement elliptique, atteignant le niveau du pharynx et recouvert à moitié par le large repli que forment les bords latéraux et postérieur du corps.

Prohemistomum syriacum Dubois, 1934, de Ciconia ciconia (L.).

Longueur: 1 mm, 26 à 1 mm, 56 .

Longueur $1^{\mathrm{mm}}, 53$ [dessin du type complété Largeur: $0 \mathrm{~mm}, 66$ à $0 \mathrm{~mm}, 93$. par celui d'un paratype].

Diamètres de la ventouse buccale: 122 à $156 \mu$ sur 90 à $135 \mu$.

Diamètres du pharynx: 105 à $120 \mu$ sur 88 à $108 \mu$.

Diamètres de la ventouse ventrale: $67 \mu$ sur $57 \mu$ (observée sur un seul exemplaire). 
La ventouse ventrale, faiblement développée et complètement cachée par l'organe tribocytique (adhésif), est située à $280 \mu$ environ de l'extrémité antérieure, c'est-à-dire aux 18/100 de la longueur du corps.

L'organe tribocytique est presque aussi large que le corps: son diamètre transversal est compris entre $54 \mu$ et $84 \mu$.

A la ventouse buccale, très grande et terminale, fait suite un pharynx ellipsoïdal et très musculeux, à la sortie duquel l'intestin bifurque immédiatement. Les caeca, larges de 80 à $90 \mu$, divergent et suivent approximativement la courbure de l'organe tribocytique pour se terminer au niveau du bord postérieur du deuxième testicule, c'est-à-dire au devant du manchon terminal que forme l'extrémité postérieure du corps.

La disposition des glandes génitales, dans la deuxième moitié du corps, est caractéristique de l'espèce: les deux testicules ovoïdes sont latéraux et obliquement opposés; l'ovaire, ovoïde ou sphérique, est situé devant le testicule postérieur, toujours un peu distant de cet organe, généralement dans la moitié droite du corps (amphitypie observée); ces deux organes ont leurs champs coincidants, mais leurs zones ne sont pas en contact.

Diamètres de l'ovaire: 160 à $245 \mu$ sur 145 à $210 \mu$.

Situation par rapport à la longueur du corps: 43/100-45/100.

Diamètres du testicule antérieur: 300 à $405 \mu$ sur 250 à $315 \mu$.

Diamètres du testicule postérieur: 270 à $430 \mu$ sur 225 à $325 \mu$.

Les glandes vitellogènes apparaissent sous forme de gros follicules ovoïdes, envahissant l'organe tribocytique et mesurant 50 à $135 \mu$ sur 30 à $100 \mu$ de diamètre. Ils sont groupés en deux masses latérales, réniformes qui s'étendent du niveau de la ventouse ventrale ou légèrement en arrière de celle-ci, c'est-à-dire des 17/100 aux 25/100 de la longueur du corps, jusqu'à la hauteur du bord postérieur du deuxième testicule, soit à l'extrémité des caeca.

La poche du cirre est longue et étroite; elle mesure $770 \mu$ à $990 \mu$ de long sur $90 \mu$ à $120 \mu$ de large. Elle s'étend jusqu'au bord antérieur de l'ovaire, de sorte que son extrémité s'observe entre les $33 / 100$ et les $45 / 100$ de la longueur du corps. Elle dépasse ainsi la moitié de l'axe longitudinal du corps et contient 
une vésicule séminale, une pars prostatica et un cirre quelquefois dévaginé.

Rapport $\frac{\text { Longueur de la poche du cirre }}{\text { Longueur du corps }}=0,58-0,65$.

L'utérus est en grande partie caché par les follicules vitellogènes. Le sphincter du vagin est bien développé: il atteint 180 à $225 \mu$ de diamètre. Les œufs, peu nombreux (2 à 17 par exemplaire), mesurent 89 à $95 \mu$ sur 60 à $67 \mu$.

Il existe actuellement 9 espèces de Prohemistomum: l'espèce type qui, d'après les recherches de AвDEL-Azim (1933), provient de Cercaria vivax Sonsino, 1892, a été décrite par Odhner, en 1913, sous le nom de Prohemistomum spinulosum Odhner. Ces 9 espèces sont:

Prohemistomum vivax (Sonsino, 1892), syn. P. spinulosum Odhner, 1913.

P. ovatus (Katsurada, 1914), syn. Paracoenogonimus ovatus Katsurada, 1914.

P. appendiculatum Ciurea, 1916.

P. industrium Tubangui, 1922.

P. odhneri Travassos, 1924.

P. serpentum Gogate, 1932.

P. fajardensis Price, 1934.

P. appendiculatoides Price, 1934.

P. joyeuxi (R. Chester Hughes, 1929) Joyeux et Baer, 1934.

De toutes ces formes, seule la quatrième, $P$. industrium Tubangui, possède un organe tribocytique (adhésif) comparable à celui de notre espèce et s'étendant presque jusqu'à l'extrémité antérieure du corps. Les différences essentielles sont les suivantes: 


\begin{tabular}{|c|c|c|}
\hline Caractères & $\begin{array}{c}\text { Prohemistomum industrium } \\
\text { Tubangui, } 1922\end{array}$ & $\begin{array}{c}\text { Prohemistomum syriacum } \\
\text { Dubois, } 1934\end{array}$ \\
\hline $\begin{array}{l}\text { Longueur } \\
\text { du corps } \\
\text { Largeur } \\
\text { maximum }\end{array}$ & $\begin{array}{l}\text { jusqu'à } 1 \mathrm{~mm}, 90 \\
1 \mathrm{~mm} \text { à } 1 \mathrm{~mm}, 2\end{array}$ & $\begin{array}{l}\text { jusqu'à } 1 \text { mm }, 56 \\
0^{\mathrm{mm}}, 66 \text { à } 0 \mathrm{~mm}, 93\end{array}$ \\
\hline $\begin{array}{l}\text { Situation du } \\
\text { repli ventral } \\
\text { formé par les } \\
\text { bords latéraux } \\
\text { et le bord } \\
\text { postérieur du } \\
\text { corps }\end{array}$ & $\begin{array}{l}\text { traverse l'axe longitudi- } \\
\text { nal du corps aux } 2 / 3 \text { de } \\
\text { sa longueur, ne recou- } \\
\text { vrant pas l'organe tribo- } \\
\text { cytique. }\end{array}$ & $\begin{array}{l}\text { traverse l'axe longitudi- } \\
\text { nal du corps au milieu } \\
\text { de sa longueur, recou- } \\
\text { vrant à moitié l'organe } \\
\text { tribocytique. }\end{array}$ \\
\hline $\begin{array}{l}\text { Diamètres: } \\
\text { Ventouse } \\
\text { buccale } \\
\text { Pharynx } \\
\text { Ventouse } \\
\text { ventrale }\end{array}$ & $\begin{array}{c}100-130 / 180-190 \mu \\
100-130 / 130-140 \mu \\
100-110 \mu\end{array}$ & $\begin{array}{c}90-135 / 122-156 \mu \\
105-120 / 88-108 \mu \\
57-67 \mu\end{array}$ \\
\hline $\begin{array}{l}\text { Testicule } \\
\text { antérieur } \\
\text { Testicule } \\
\text { postérieur }\end{array}$ & $\begin{array}{l}\text { médians; placés l'un der- } \\
\text { rière l'autre, à champs } \\
\text { coïncidants et à zones en } \\
\text { contact; le point de con- } \\
\text { tact est situé environ à mi- } \\
\text { distance entre l'extrémité } \\
\text { antérieure et l'extrémité } \\
\text { postérieure du corps. } \\
\quad 490-520 / 330-450 \mu \\
650-810 / 360-380 \mu\end{array}$ & $\begin{array}{l}\text { latéraux; opposés obli- } \\
\text { quement, à champs non } \\
\text { coïncidants et à zones im- } \\
\text { briquées; toujours situés } \\
\text { dans la seconde moitié } \\
\text { du corps. } \\
\quad 300-405 / 250-315 \mu \\
270-430 / 225-325 \mu\end{array}$ \\
\hline $\begin{array}{l}\text { Ovaire } \\
\text { Situation }\end{array}$ & $\begin{array}{l}\quad 150-190 \mu \\
\text { à côté de la ligne média- } \\
\text { ne; ventralement et au } \\
\text { niveau de la moitié pos- } \\
\text { térieure du premier testi- } \\
\text { cule; opposé à la poche } \\
\text { du cirre. }\end{array}$ & $\begin{array}{l}160-245 / 145-210 \mu \\
\text { latéralement; en avant } \\
\text { du testicule postérieur et } \\
\text { opposé obliquement au } \\
\text { testicule antérieur. }\end{array}$ \\
\hline CEufs & $130-146 / 89-97 \mu$ & $89-95 / 60-67 \mu$ \\
\hline Hôte & Chien & Cigogne \\
\hline
\end{tabular}


Collections. - Le type et les paratypes (du tube $n^{0} 240$ ) sont déposés dans notre collection particulière.

Les exemplaires provenant du tube no 241 sont déposés dans la Collection du Département de Parasitologie de l'Université Hébraïque, à Jérusalem̀n.

\section{BIBLIOGRAPHIE}

1933. Aвdel Azim, M. On Prohemistomum vivax (Sonsino, 1892) and its development from Cercaria vivax Sonsino, 1892. Zeitschr. f. Parasit., vol. V, p. 432-436.

1916. Crunea, J. Prohemistomum appendiculatum, eine neue Holostomiden-Art aus Hunden- und Katzendarm, dessen Infektionsquelle in den Süsswasserfischen zu suchen ist. Zeitschr. Infektionskrankh. Parasit. Krankh. u. Hyg. d. Haustiere, Bd. XVII, p. 309-328, Taf. XV-XVI.

1927. Contribution à l'étude morphologique de Strigea cornu (Rud.). Bull. Sect. scient. Acad. Roumaine, vol. XI, p. 12-16, pl. I-II, 5 fig.

1932. Dubois, G. Revision des Hémistomes et étude de formes nouvelles. Bull. Soc. neuch. Sc. nat., Neuchâtel, t. LVI (n. s., t. V), p. 375-412, 5 fig., pl. I-IV.

1934. - Nouveaux parasites d'Oiseaux. Actes Soc. Helv. des Sc. nat., Zurich, 1934, p. 374-375.

1932. Gogate, B. S. On a new species of Trematode (Prohemistomum serpentum n. sp.) from a snake, with a note on an immature species of Heterechinostomum Odhner from the cat. Parasitol., vol. XXIV, p. 318-320, 2 fig.

1929. Hughes, R. C. Studies on the Trematode family Strigeidae (Holostomidae). No. XIV: Two new species of Diplostomula. Occas. papers of the Mus. Zool. Univ. of Michigan, no 202, p. 1-28, pl. I.

1934. Joyeux, Ch. et Baer, J.-G. Sur un Trématode de Conleure. Rev. suisse Zool., t. XLI, p. 203-215, 4 fig.

1914. Katsurada, F. Studien über Trematodenlarven bei Süsswasserfischen, mit besonderer Berücksichtigung der Elb- und Alsterfische (vorl. Mitt.). Centralbl. f. Bakt., Parasitenk. u. Infektionskrankh.Orig., Bd. 73, p. 304-314, 9 Textfig., 1 Taf. 
1926. La Rue, G. R. Studies on the Trematode family Strigeidae (Holostomidae). No. II: Taxonomy. Trans. Amer. Micr. Soc., vol. XLV, p. 11-19.

1913. Odhner, T. Zum natürlichen System der digenen Trematoden. VI. Zool. Anz., Bd. XLII, p. 289-318, 13 Fig.

1934. Price, E. W. New Trematode Parasites of Birds. Smithsonian Miscellaneous Coll., vol. 91, p. 1-6, pl. I.

1925. Poche, F. Das System der Platodaria. Arch. f. Naturgesch., Berlin, Jahrg. XCI A, Hefte 2/3, p. 1-458, 7 Taf., 16 Fig.

1929. Szidat, L. Beiträge zur Kenntnis der Gattung Strigea (Abildg.). I: Allgemeiner Teil. II : Spezieller Teil. Zeitschr. f. Parasit. Bd. 1, p. 612-764, 70 Fig., 1 Taf.

1924. Travassos, L. Nouvelle espèce de Prohemistomum. C.R. Soc. Biol., Paris, t. XCI, p. 835-838, 3 fig.

1922. Tubangui, M. A. Two new intestinal Trematodes from the Dog in China. Proc. Unit. States Nat. Mus., vol. LX, p. 1-12, pl. 1-4. 


\section{$2 \mathrm{BHL}$ Biodiversity Heritage Library}

Dubois, Georges. 1935. "Etude des Strigéidés de la collection du département de Parasitologie de l'Université Hébraïque de Jérusalem." Revue suisse de zoologie 42, 571-585. https://doi.org/10.5962/bhl.part.117668.

View This Item Online: https://www.biodiversitylibrary.org/item/148565

DOI: https://doi.org/10.5962/bhl.part.117668

Permalink: https://www.biodiversitylibrary.org/partpdf/117668

\section{Holding Institution}

American Museum of Natural History Library

\section{Sponsored by}

BHL-SIL-FEDLINK

\section{Copyright \& Reuse}

Copyright Status: In copyright. Digitized with the permission of the rights holder.

Rights Holder: Muséum d'histoire naturelle - Ville de Genève

This document was created from content at the Biodiversity Heritage Library, the world's largest open access digital library for biodiversity literature and archives. Visit BHL at https://www.biodiversitylibrary.org. 